\title{
Myelomatous Pleural Effusion Presenting with Extreme Hyperferritinemia and Severe Inflammatory Response
}

\author{
Diego A. Adrianzen Herrera ${ }^{a} \quad$ Matthew Scott $^{b} \quad$ Mala Varma $^{c}$ \\ ${ }^{a}$ Icahn School of Medicine at Mount Sinai, St. Luke's-Roosevelt Hospital Center Residency \\ program, ${ }^{b}$ Department of Medicine and ${ }^{\mathrm{C}}$ Division of Hematology/Oncology, Department \\ of Medicine, Mount Sinai St. Luke's-Roosevelt Hospital, New York, N.Y., USA
}

\section{Key Words}

Multiple myeloma $\cdot$ Pleural effusion $\cdot$ Hyperferritinemia

\begin{abstract}
Myelomatous involvement of pleural effusions developing in patients with multiple myeloma is extremely rare and only a few cases have been reported so far. It is thought to represent an aggressive clinical progression of disease and is usually associated with severe complications, poor prognosis and high mortality. Ferritin is a marker of inflammatory pathways that plays a significant role in plasma cell malignancies and has been studied as a prognostic factor for multiple myeloma. In severe inflammatory states such as septic shock or hemophagocytic lymphohistiocytosis, extreme levels of ferritin are thought to precipitate a cytokine storm associated with poor clinical outcomes. We present a case of myelomatous pleural effusion associated with extreme levels of ferritin and explore the possibility of a connection between this rare entity and other severe inflammatory states, which could account for its ominous outcomes and poor prognosis.

\section{Introduction}

Multiple myeloma (MM) is a common disease, accounting for $10 \%$ of hematologic malignancies [1]. Pleural effusions occur in up to $6 \%$ of patients with MM [1,2] but rarely as a result of primary myelomatous infiltration or pleural involvement [2-4]. Most pleural effu-

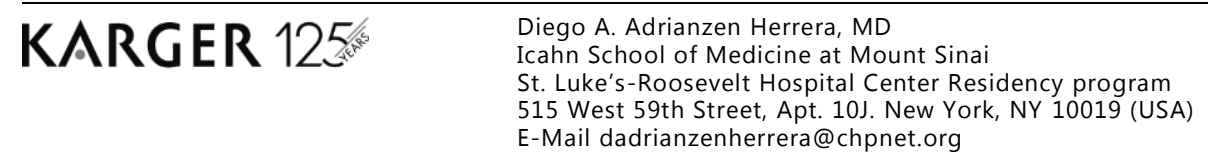


Adrianzen Herrera et al:: Myelomatous Pleural Effusion Presenting with Extreme Hyperferritinemia and Severe Inflammatory Response

sions in MM are secondary to heart failure from amyloidosis, nephrotic or nephritic syndrome, pulmonary embolism, lymphatic obstruction, hypoalbuminemia or infections $[3,5$, 6]. Myelomatous pleural effusion (MPE) is a rare entity, occurring in only about $1 \%$ of MM pleural effusions [1-5] with $<100$ having been reported [6]. MPE is usually a sign of advanced disease [1, 2, 7] and the reported median survival time is $<4$ months [5].

Diagnosis is based on criteria proposed by the International Myeloma Working Group [8], and includes monoclonal protein detection by pleural fluid protein electrophoresis, presence of atypical plasma cells in the effusion or histological confirmation by pleural biopsy $[3,7]$. The most frequently used study is pleural cytology [1, 2, 9-11], which establishes diagnosis in $>90 \%$ of cases [5] and has been considered the best method [1], although electrophoresis confirmation is sometimes advocated for $[7,12]$.

A general male predominance has been found [1,2], and associated pleural or chest wall plasmacytomas are usually present $[1,4]$. Although its development in advanced disease is more common [2-4, 6, 7, 9], less frequently it is the initial presenting feature of MM $[1,5]$. The most common isotype associated with MPE is unclear. A higher prevalence of IgG and IgA was found in different series $[1,2,4]$, while light chain type is uncommon $[2,4]$ except in MPE as initial presentation [1].

Ferritin is irregularly used as a tumor marker and has recently been explored as a prognostic factor [10] in MM, as it can reliably represent the inflammatory and oxidant pathways that play a significant role in plasma cell malignancies [11]. We describe a case in which MPE presented with extremely high ferritin levels, reflecting the potential value of ferritin as an MM marker.

\section{Case Presentation}

A 40-year-old male was admitted to our institution for acute hypoxic respiratory failure for 3 days. Seven months prior to admission, he had been diagnosed with MM stage III (according to the International Staging System). At that time, records showed a bone marrow (BM) biopsy consistent with plasma cell myeloma: hypercellular marrow (90\%) with residual trilineage hematopoiesis and increased immature morphology plasma cell infiltrate involving $60-70 \%$ of cellularity. Flow cytometry of BM specimen showed a clonal population of plasma cells (27\%) with an immunophenotype positive for CD138, CD38 and CD56 markers. Flow cytometry of peripheral blood exhibited 5\% circulating myeloma cells. Cytogenetics displayed complex abnormalities consisting of monosomy 13, trisomy 3 and 9 and $1 p$ deletion. Serum protein electrophoresis showed a monoclonal IgA lambda paraprotein and urine protein electrophoresis revealed a monoclonal lambda light chain (Bence-Jones protein). An abdominal wall fat pad biopsy was negative for amyloid. Imaging studies revealed widespread lytic lesions in the calvarium, bilateral humerus, pelvis and spine, as well as a $5.8 \times 3.8 \mathrm{~cm}$ right 5 th lateral rib plasmacytoma.

At the time of the initial diagnosis, he showed high disease activity with a baseline $\beta_{2^{-}}$ microglobulin level of $6.2 \mathrm{mg} / \mathrm{l}$ and a serum ferritin level of $728 \mathrm{ng} / \mathrm{ml}$. He was also found to have compromised kidney function consistent with stage III chronic kidney disease from light chain nephropathy, but no major hepatic, pulmonary or coagulation abnormalities were found. He failed treatment with vincristine, cyclophosphamide, and methylprednisolone as well as bortezomib, cyclophosphamide, and Solu-Medrol, but remarkably responded to a modified phase II study regimen with bortezomid, bendamustine, and dexamethasone for refractory MM. His last cycle was completed 10 days before presentation to our institution. 
Adrianzen Herrera et al.: Myelomatous Pleural Effusion Presenting with Extreme Hyperferritinemia and Severe Inflammatory Response

Upon admission, he required noninvasive mechanical ventilation and high-dose supplemental oxygen. After ruling out pulmonary embolism with a ventilation/perfusion scan and heart failure with a normal brain natriuretic peptide level and left ventricular function on the echocardiogram, infectious causes were considered, despite normal body temperature, as chest imaging showed bilateral ground-glass opacities with superimposed interlobular septal thickening and intralobular reticular thickening (fig. 1). He received empiric broad-spectrum antibiotics, including coverage for atypical bacteria and Pneumocystis jiroveci. Serial blood cultures were negative for fungal and bacterial pathogens. He underwent bronchoscopy and had negative fungal and bacterial bronchoalveolar lavage cultures. Cytology only revealed nonspecific pigmented macrophages. Bronchoalveolar lavage with methenamine silver stain for fungal and $P$. jiroveci-like organisms was negative, as were immunohistochemical studies for cytomegalovirus and herpes simplex virus. Special stains including Congo red and periodic acid-Schiff stain were negative for amyloidosis and pulmonary alveolar proteinosis, respectively.

After a brief initial response to supportive respiratory measures and empiric antimicrobial therapy, he developed multisystemic organ failure, including lactic acidosis, acute liver failure with metabolic encephalopathy, acute kidney injury and worsening pancytopenia dependent on blood and platelet transfusions. The workup revealed active myelomatous disease with rising serum IgA and $\beta_{2}$-microglobulin levels to $8.6 \mathrm{mg} / \mathrm{l}$. Remarkably, a serum ferritin level of $11,598.5 \mathrm{ng} / \mathrm{ml}$ was found and concern for hemophagocytic lymphohistiocytosis (HLH) led to empiric treatment with high doses of dexamethasone and an urgent BM biopsy, which showed atypical plasma cells comprising $90 \%$ of the marrow cellularity but no evidence of hemophagocytosis or increased blasts.

Sequential chest imaging showed a timely correlation between his acute clinical decline and the acute development of a large right pleural effusion. Thoracentesis revealed a heavily blood stain exudate $(\mathrm{pH} 7.38$, protein $6.9 \mathrm{~g} / \mathrm{dl}$, lactate dehydrogenase 1,543 IU/l, white

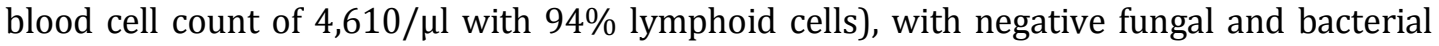
cultures. Cytology showed atypical plasma cell proliferation (fig. 2) and immunostaining confirmed MM involving pleural effusion [CD138- and CD38-positive (fig. 3) with negative MOC31, BerEP4 and CD45 markers]. High-dose steroids were continued and the effusion was drained; however, multisystemic organ failure prevented the initiation of intravenous chemotherapy. Despite aggressive supportive measures, he developed disseminated intravascular coagulation, required endotracheal intubation, vasopressor support, and finally perished soon after the diagnosis.

\section{Discussion}

Myelomatous involvement of extramedullary sites can lead to various clinical presentations in MM [7]. MPE is extremely rare, with a recent series reporting only 64 cases from relevant sources in the English literature since 2000 [1]. We present a case of MPE associated with hyperferritinemia and severe multiorgan failure, a clinical course resembling catastrophic inflammatory conditions such as HLH that has not been described in MPE before.

Given its infrequency, a high suspicion is needed for diagnosis [7]. Only about $1 \%$ of pleural effusions in MM patients will be attributed to myelomatous involvement [1-5], but unless the clinical presentation is obvious and rules it out, we believe MPE should always be considered in MM patients presenting with pleural effusions. This is important because MPE represents an aggressive clinical progression of MM, and a recent series reported a median survival of 2.8 months despite aggressive local and systemic treatment [2]. As in our case, 
Adrianzen Herrera et al.: Myelomatous Pleural Effusion Presenting with Extreme Hyperferritinemia and Severe Inflammatory Response

the diagnosis is most likely revealed by pleural cytology $[1,2,5]$. Protein electrophoresis is another option [8], by itself or as a confirmation to cytology [6], but is unreliable since most effusions are hemorrhagic [2]. Pleural biopsies are less commonly used due to their inconvenience [7] and a lower diagnostic yield given the patchy pleural involvement in MM [5].

Some risk factors for developing MPE in MM were found in our patient and are consistent with the review of other reported cases and series. These include its occurrence in the late stage of MM $[2,4,7]$, association with high-risk cytogenetics, such as deletion 13 chromosomal abnormality [1,2] and usual coexistence of chest wall plasmacytomas [1, 4]. The MM subtype does not seem to be a risk factor given that IgG was most common in 2 series with a total of 28 patients [1, 4], while IgA was predominant in a 19-patient singleinstitution series [2] and overall all types have been reported in at least 1 case [7-9]. Except in MPE as initial presentation of MM, where it constituted 24\% of the cases [1], light chain type seems to be very uncommon $[2,4]$.

As the majority of the literature on this topic is from single reported cases and small series, the relationship between tests or treatment and prognosis is not known; however, as prognosis is known to be poor, palliation is considered the mainstay of treatment and includes chemotherapy and pleurodesis [1]. There is no chemotherapeutic regimen of choice since a wide variety has been used over time and no meaningful comparisons can be made $[1,2,4]$.

Recent studies suggest that the inflammatory and oxidant pathways play a significant role in the pathogenesis of plasma cell malignancies, and ferritin levels can be a reliable indicator of these processes in carcinogenesis [10]. Therefore, ferritin has been used as a marker in MM and other hematologic malignancies $[10,11]$. Studies show that ferritin levels are an independent predictor of mortality in newly diagnosed MM [12], that higher ferritin levels are present in recurrent MM compared to patients in remission [11] and that ferritin has a prognostic value in MM patients undergoing autologous transplantation [13, 14]. This evidence has been proposed to reflect a correlation between ferritin levels and disease activity, tumor load and malignant potential [14].

Our case is the first report that links MPE to extreme levels of hyperferritinemia, which are usually present only in medical conditions associated with severe inflammatory states such as HLH and septic shock where ferritin is thought to have a pathogenic role leading to a cytokine storm and catastrophic clinical outcomes known as the hyperferritinemic syndrome [15]. It is known that hematologic malignancies and sepsis can lead to HLH, but this was ruled out in our patient. Furthermore, no infectious source was ever found despite extensive workup. Unfortunately, ferritin levels have not been reported in previous MPE cases, and it is unknown if this finding is an isolated coincidence or if it has been missed in previous reports. We believe MPE could represent or be part of an extreme manifestation of myeloma disease activity leading to a severe inflammatory cascade with subsequent multiorgan failure. We recommend investigating ferritin levels in patients with MPE to better determine its relationship with pathogenic inflammatory states such as HLH. This relationship could certainly explain the poor prognosis and severe clinical complications seen with MPE.

\section{Statement of Ethics}

Written informed consent for personal clinical and imaging information was obtained from the patient before publication of this case report and its accompanying images. A copy of the written consent is available for review from the Editor-in-Chief of this journal. 


\section{Case Reports in Oncology}

\begin{tabular}{l|l}
\hline Case Rep Oncol 2015;8:349-355 \\
\hline DOI: $10.1159 / 000438983$ & $\begin{array}{l}\text { C } 2015 \text { The Author(s). Published by S. Karger AG, Basel } \\
\text { www.karger.com/cro }\end{array}$ \\
\hline
\end{tabular}

Adrianzen Herrera et al:: Myelomatous Pleural Effusion Presenting with Extreme Hyperferritinemia and Severe Inflammatory Response

\section{Disclosure Statement}

The authors declare that there is no conflict of interest regarding the publication of this paper.

\section{References}

-1 Zhang LL, Li YY, Hu CP, Yang HP: Myelomatous pleural effusion as an initial sign of multiple myeloma - a case report and review of the literature. J Thorac Dis 2014;6:E152-E159.

$>2$ Cho YU, Chi HS, Park CJ, Jang S, Seo EJ, Suh C: Myelomatous pleural effusion: a case series in a single institution and literature review. Korean J Lab Med 2011;31:225-230.

-3 Tsukamoto A, Yoshiki Y, Yamazaki S, Kumano K, Nakamura F, Kurokawa M: The significance of free light chain measurements in the diagnosis of myelomatous pleural effusions. Ann Hematol 2014;93:507-508.

4 Kamble R, Wilson S, Fassas A, et al: Malignant pleural effusion of multiple myeloma: prognostic factors and outcomes. Leuk Lymphoma 2005;46:1137-1142.

5 Alshati M, Kumar R, Kannan S: Dyspnea, massive effusion and lytic rib lesion as initial presentation of multiple myeloma in a young man. Can Respir J 2013;20:253-255.

-6 Oudart, JB, Maquart FX, Semouma 0, Lauer M, Arthuis-Demoulin P, Ramont L: Pleural effusion in a patient with multiple myeloma. Clin Chem 2012;58:672-674.

7 Al-Farsi K, Al-Haddabi I, Al-Riyami N, Al-Sukaiti R, Al-Kindi S: Myelomatous pleural effusion. Case report and review of the literature. Sutan Qaboos Univ Med J 2011;11:259-264.

8 International Myeloma Working Group: Criteria for the classification of monoclonal gammopathies, multiple myeloma and related disorders: a report of the International Myeloma Working Group. Br J Haematol 2003;121:749-757.

-9 Huang TC, Chao TY: Myelomatous pleural effusion. QJM 2010;103:705-706.

10 Lodh M, Goswami B, Gupta N, Patra S, Saxena A: Assessment of oxidative stress and inflammatory process in patients with multiple myeloma. Ind J Clin Biochem 2012;24:410-413.

11 Zhang XZ, Su AL, Hu MQ, Zhang XQ, Xu YL: Elevated serum ferritin levels in patients with hematologic malignancies. Asian Pac J Cancer Prev 2014;15:6099-6101.

12 Song MK, Chung JS, Seol YM, Shin HJ, Choi YJ, Cho GJ: Elevation of serum ferritin is associated with the outcome of patients with newly diagnosed multiple myeloma. Korean J Intern Med 2009;24:368-373.

13 Strasser-Weippl K, Ludwig H: Ferritin as prognostic marker in multiple myeloma patients undergoing autologous transplantation. Leuk Lymphoma 2014;55:2520-2524.

14 Shimoni A: Risk stratification of patients with multiple myeloma prior to autologous stem cell transplant: what is the role of serum ferritin levels? Leuk Lymphoma 2014;55:2419-2420.

15 Rosario C, Zandman-Goddard G, Meyron-Holtz EG, D’Cruz DP, Shoenfeld Y: The hyperferritinemic syndrome: macrophage activation syndrome, Still's disease, septic shock and catastrophic antiphospholipid syndrome. BMC Med 2013;11:185. 
Adrianzen Herrera et al.: Myelomatous Pleural Effusion Presenting with Extreme Hyperferritinemia and Severe Inflammatory Response

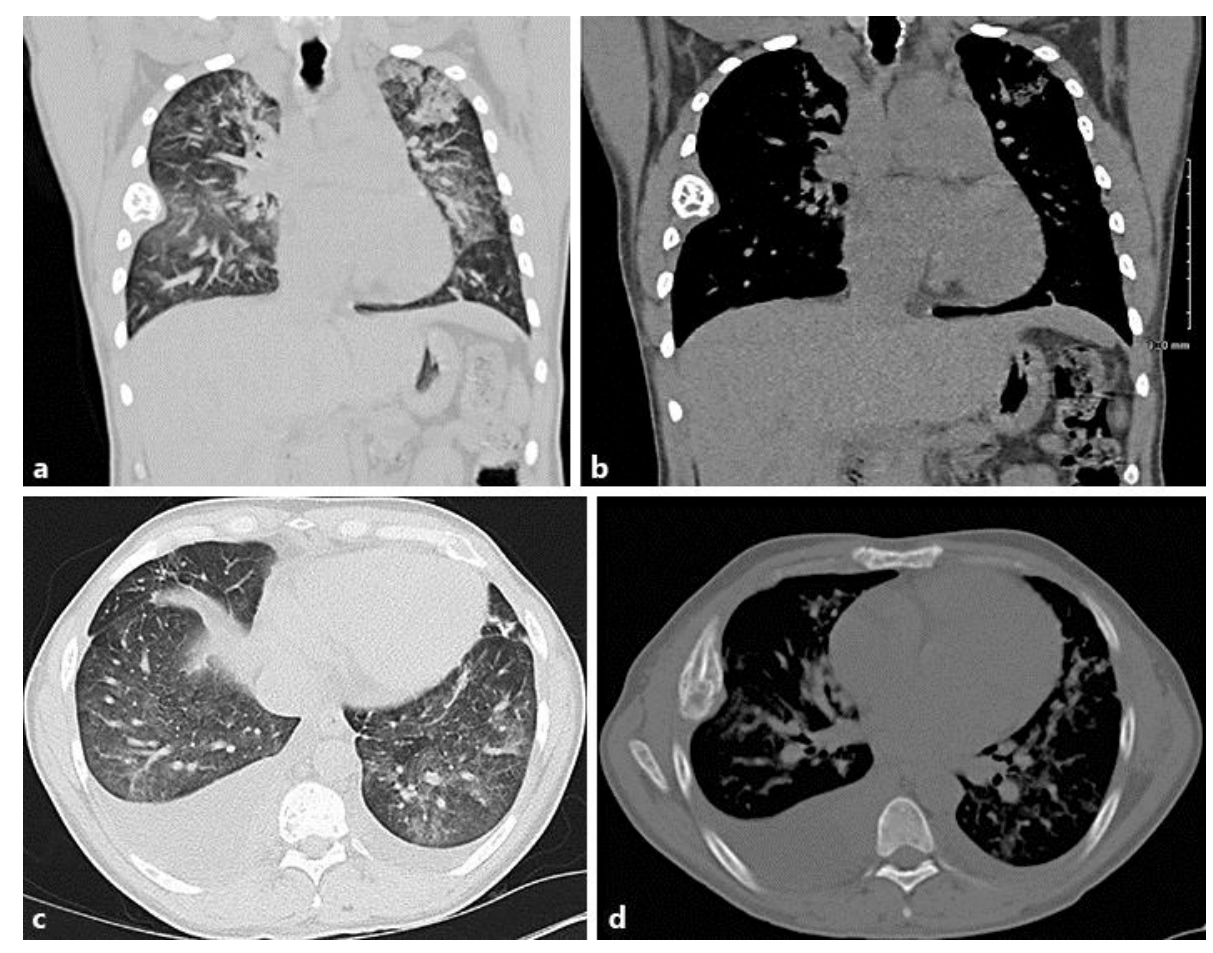

Fig. 1. a, b CT scan of the chest showing a coronal cut of the 5th rib plasmacytoma. a Pulmonary view. b Mediastinal view. c, d Large pleural effusion. c Pulmonary view. d Mediastinal view. Note the bilateral ground-glass opacity which led to the initial consideration of infectious causes.

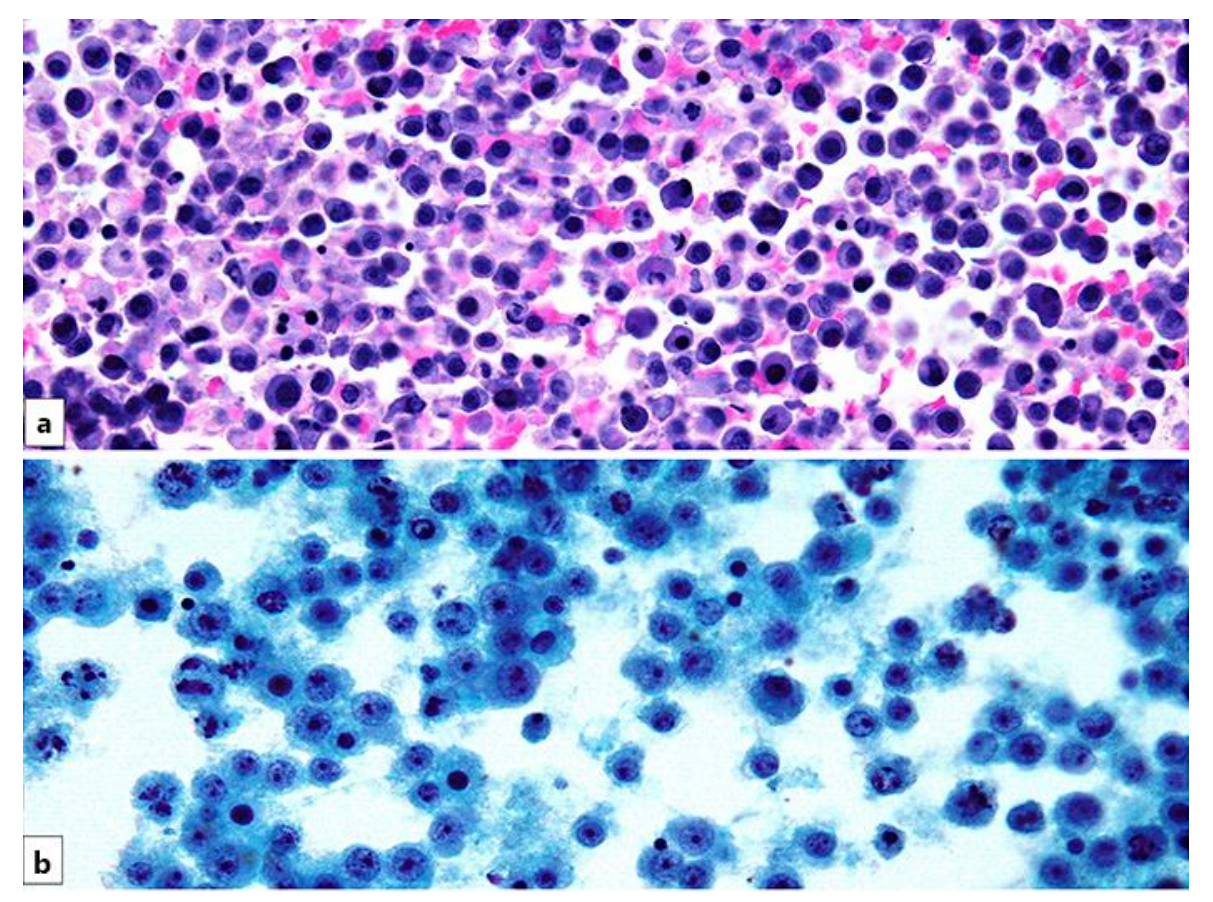

Fig. 2. Pleural effusion cytology. $\times 40$. Cell block stained with hematoxylin stain (a) and Papanicolaou stain (b). 
Adrianzen Herrera et al.: Myelomatous Pleural Effusion Presenting with Extreme Hyperferritinemia and Severe Inflammatory Response

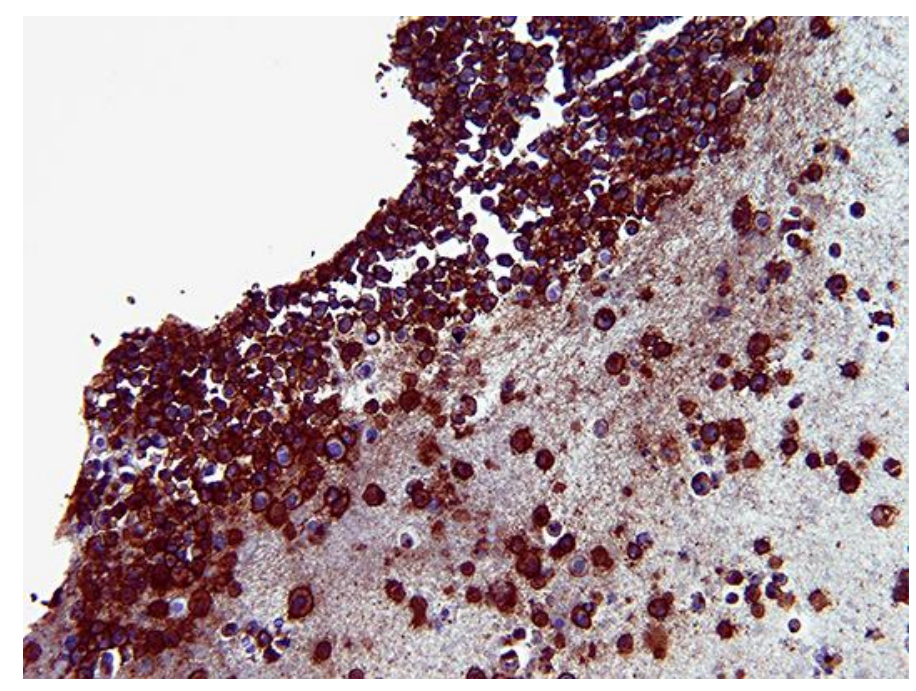

Fig. 3. CD138 (syndecan-1) stain in pleural effusion. $\times 40$. 